\title{
HOLZFORSCHUNG
}

MITTEILUNGEN ZUR CHEMIE, PHYSIK, BIOLOGIE UND TECHNOLOGIE DES HOLZES

2I. Band - Heft s

TECHNISCHER VERLAG HERBERT CRAM, BERLIN 30

HERAUSGEBER DR. G. STEGMANN, BRAUNSCHWEIG

OKTOBER 1967

\section{Untersuchungen über die Strahlenresistenz holzzerstörender Pilze}

\author{
Von J. Bors und H. Glubrecht
}

Institut für Strahlenbiologie der Technischen Hochschule Hannover

\section{Problemstellung}

Nach wie vor hat das Problem der Beseitigung von Holzschäden, die durch Pilze verursacht werden, nichts an Aktualität verloren. Besonders nach dem Krieg wurde durch die Zerstörung einzelner Gebäudeteile die Verbreitung holzzerstörender Pilze begünstigt. Überschwemmungen und Grundwasser sorgen noch immer für genügend Feuchtigkeit, die für die Verbreitung der Pilze mitverantwortlich ist. Diesem Faktor kommt besondere Bedeutung in Neubauten zu, wenn nicht hinreichend ausgetrocknetes Holz eingebaut wird. Die zerstörende Tätigkeit der Pilze beschränkt sich keineswegs auf das in herkömmlicher Weise eingebaute Holz, sondern es wird auch das in der modernen Holztechnologie benutzte $\mathrm{Holz}$, z. B. das mineralisch gebundene, angegriffen (Künzelmann I96I).

Für die direkte Bekämpfung holzzerstörender Pilze stehen zur Zeit chemische Mittel zur Verfügung, deren Wirkung durch bautechnisch-physikalische Maßnahmen noch erhöht wird. Einige Probleme treten jedoch bei dieser Bekämpfungsmethode auf. Die chemischen Mittel werden mit der Zeit abgebaut und verlieren ihre Wirksamkeit. Vor allem ist es schwer sicherzustellen, daß alle irgendwo im Gebäude verteilten Reste von Pilzmaterial oder Sporen von den eingesetzten Chemikalien erreicht werden. In jedem Falle sind mehr oder weniger umfangreiche bauliche Maßnahmen bei der Bekämpfung erforderlich. Meistens sind die Gebäude für längere Zeit unbewohnbar. Bei kunstgeschichtlich wertvollen Gebäuden können bauliche Veränderungen problematisch sein, und außerdem muß durch die Einwirkung von Chemikalien eine Wertminderung befürchtet werden.

Die Frage der Strahlenempfindlichkeit von Bakterien und Pilzen ist ursprünglich vorwiegend unter Gesichtspunkten der Grundlagenforschung in der Strahlenbiologie behandelt worden. Seit einigen Jahren stehen auch praktische Anwendungsfragen im Zusammenhang mit der Lebensmittelkonservierung bei solchen Untersuchungen im Vordergrund (IAEA Symp., Karlsruhe I966). Es liegt nahe, derartige Untersuchungen auch auf die holzzerstörenden Pilze auszudehnen, um festzustellen, ob eine Bekämpfung mit Hilfe ionisierender Strahlen möglich ist. Zur Zeit stehen zwar vereinzelt Angaben über die Strahlenresistenz dieser Pilze zur Verfügung, jedoch wurde daraus bisher kein praktischer Nutzen gezogen. So bringt Babos (I962) einige Daten über die Strahlenresistenz des Mycels holzzerstörender
Pilzarten. Seine Untersuchungen waren hauptsächlich informativer Art. Die von ihm erreichten Letaldosen decken sich nicht mit den Dosen, die Frejdin, Gorsin und Krapivna (I96I) ermittelt haben. Frejdin und Mitarbeiter untersuchten einzelne Pilzarten der Klassen der Ascomyceten, Basidiomyceten und der Fungi imperfecti. Sie fanden heraus, daß gefährliche Holzzerstörer wie Coniophora cerebella, Poria vaporaria und Merulius pinastri ihre Lebensfähigkeit bei Strahlendosen von etwa 200 krad verlieren. Diese Dosen liegen weit unter denen, die das Holz nachteilig beeinflussen. Daß Frejdin und Mitarbeiter Merulius pinastri statt $M$. lacrymans untersuchten, liegt vermutlich an der geographischen Verbreitung dieser Arten. In Westund Mitteleuropa ist Merulius lacrymans, der echte Hausschwamm, der meistverbreitete und gefährlichste Bauholzzerstörer. Daher ist die Untersuchung seiner Strahlenresistenz vordringlich. Gerade in Hinblick auf die praktische Anwendung ist von vornherein der Einfluß von Parametern zu berücksichtigèn, die die Strahlenempfindlichkeit herauf- oder herabsetzen können. Empfindlichkeitssteigernd wirken bekanntlich in vielen Fällen Temperaturerhöhung und $\mathrm{O}_{2}-$ Überschuß. Da die Erhöhung der Temperatur in Gebäuden keinen großen technischen Aufwand erfordert, wurde zunächst mit diesem sensibilisierenden Faktor gearbeitet. Als Folge der Bestrahlung wird häufig das physiologische Gleichgewicht gestört, und so ist naheliegend, daß die Pilze je nach Nährsubstrat unterschiedlich auf die Bestrahlung reagieren. Deshalb wurden sie für die Bestrahlung vergleichsweise im Biomalz-Agar und im Holz vorkultiviert.

Über die Bestrahlung von Merulius-Sporen liegt zur Zeit keine Arbeit vor. Die Sporen von Merulius sind hauptsächlich für die Verbreitung des Pilzes über größere Entfernungen verantwortlich. So wurden sie neben dem Mycel im Hinblick auf ihre Strahlenresistenz untersucht.

Bei den Arbeiten tauchten einige Fragen auf, deren Lösung einerseits für die praktische Bekämpfung erforderlich und andererseits von allgemeinem biologischen Interesse war. Über die hierbei gewonnenen Erfahrungen wird ebenfalls berichtet.

Als weiterer gefährlicher Holzzerstörer wurde Coniophora cerebella, der Kellerschwamm, der seine destruktive Tätigkeit besonders bei höherer relativer Luftfeuchtigkeit ausübt, in die Untersuchung mit einbezogen. 


\section{Material und Methode}

Als Untersuchungsmaterial dienten die Reinkulturen von Merulius lacrymans (Jacq.) Fr. und Coniophora cerebella (Pers.) Duby. Sie wurden vom "Centraalbureau voor Schimmelcultures - Baarn" bezogen und als Schrägkulturen auf BiomalzAgar weiterkultiviert. In neuen taxonomischen Arbeiten wird Merulius lacrymans unter dem Namen Serpula lacrimans geführt, doch erscheint eine Beibehaltung der älteren Bezeichnung als zweckmäßig, da sie wesentlich weiter verbreitet sein dürfte (Kreisel I96I).

Proben für die Bestrahlung des Mycels im Biomalz-Agar wurden aus Petrischalenkulturen gewonnen. Der Nährboden setzte sich aus $40 \mathrm{~g}$ Biomalz und $20 \mathrm{~g}$ Agar-Agar je $1000 \mathrm{ml}$ $\mathrm{H}_{2} \mathrm{O}$ zusammen. Sobald die ganze Fläche von der Mitte der Petrischale aus mit Oberflächenmycel durchwachsen war, wie dies auch die Abb. I veranschaulicht, wurden für die $\mathrm{Be}-$ strahlung kreisförmige Stücke mit einem Durchmesser von I cm mit einem einfachen Korkbohrer ausgestanzt.

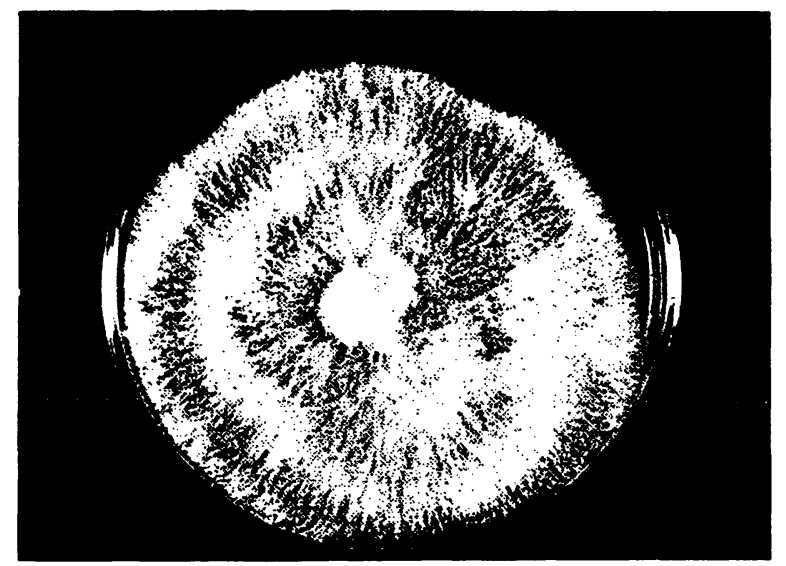

Abb. I. Oberflächenmycel von Merulius lacrymans auf BiomalzAgar in Petrischale

Infizierte Holzproben wurden in der Weise gewonnen, indem ro $\mathrm{cm}$ lange Kiefernholzstücke mit einer Grundfläche von $2 \times I \mathrm{~cm}$ auf Schrägagarkulturen in $30 \mathrm{~mm}$ Reagenzgläsern dem Mycelangriff ausgesetzt waren. Diese Methode zeigt die Abb. 2 mit Merulius lacrymans.

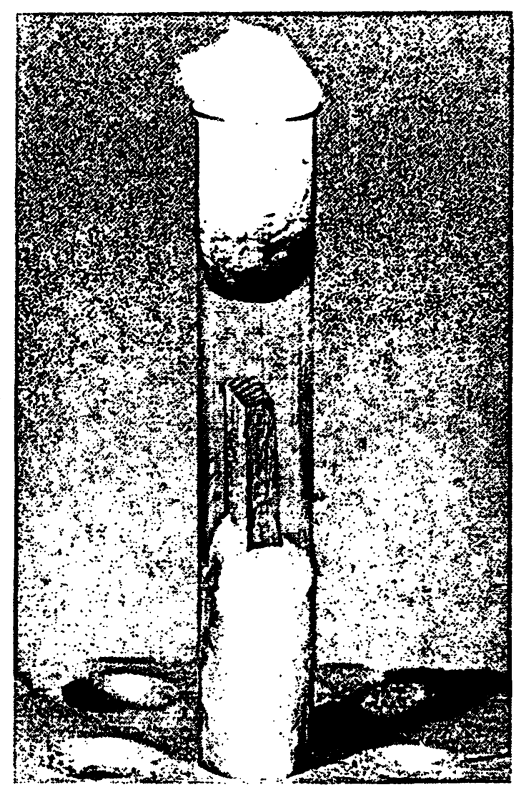

Abb. 2. Mycelwachstum von Merulius auf Holz im Reagenzglas

Nach einer Pilzeinwirkung von mindestens 4 Wochen wurden diese Holzproben in Stücke von $\mathrm{I} \mathrm{cm}$ Länge und $2 \times 3 \mathrm{~mm}$ Breite zerlegt und dann in speziellen Küvetten bestrahlt. Die
Größenabmessung der Impfstücke war durch bestrahlungstechnische Gründe bedingt. Außerdem konnte die Dosimetrie auf diese Weise mit sehr genauer Lokalisierung durchgeführt werden.

Als Strahlungsquelle diente ein ${ }^{137} \mathrm{Cs}$-Präparat mit einer Aktivität von Io Ci. Die Dosisleistung betrug in unserer Anordnung $210 \mathrm{rad} / \mathrm{min}$. Die Proben eines Versuches erhielten eine simultane Temperaturbehandlung während der Bestrahlung. Die Bestrahlungsanordnung ist auf Abb. 3 schematisch dargestellt.

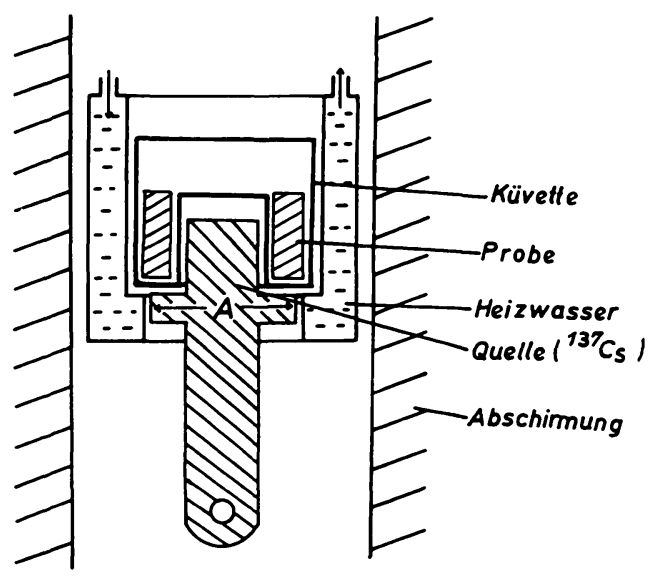

$$
A=10,0 \mathrm{~mm}
$$

Abb. 3. Anordnung zur Bestrahlung von Pilzkulturen

Die jeweils in zehnfacher Wiederholung bestrahlten Proben wurden dann auf Agar-Nährboden in Petrischalen gelegt, und als Kriterium der Strahlenwirkung wurde das Längenwachstum des Mycels nach $7 \mathrm{bzw}$. ro Tagen Kultur bei $18^{\circ} \mathrm{C}$ in $\mathrm{mm}$ gemessen. Die Abb. 4 zeigt eine Petrischale mit weißem Oberflächenmycel von Merulius mit Holzproben.

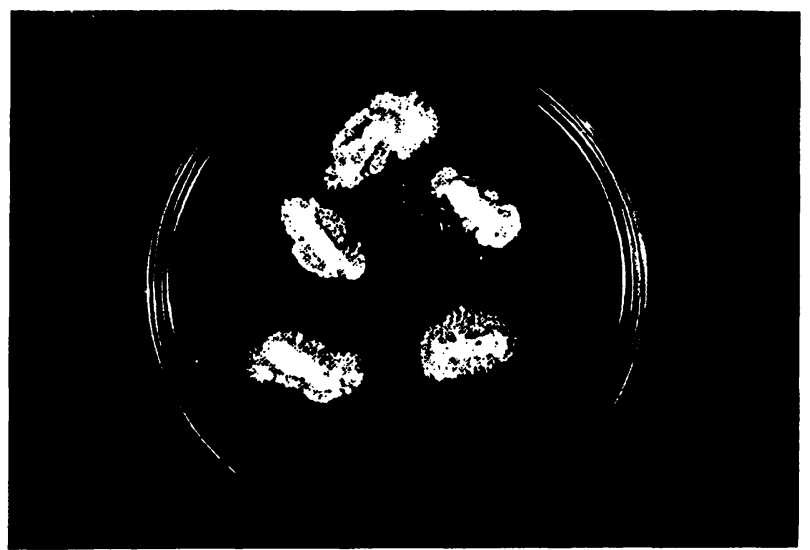

Abb. 4. Mycelwachstum aus den Holzproben in einer Petrischale

Eine andere Möglichkeit der Charakterisierung der Strahlenwirkung wurde ebenfalls erprobt. Hierbei legten wir die Impfstücke in rooer Erlenmeyerkolben in eine Nährlösung nach Lyr (Lyr 1959). Nach 30 Tagen Kultur bestimmten wir das Trockengewicht. In der ermittelten Trockensubstanz traten jedoch, vermutlich aus impftechnischen Gründen, zu große Schwankungen auf, so daß mit dieser Methode nicht weiter gearbeitet wurde. Außerdem erschien die Wachstumszeit von 30 Tagen als reichlich lang. Eine Verkürzung dieser Zeit durch Schütteln der Kulturen konnte nicht erreicht werden.

Zur Gewinnung der Sporen von Merulius lacrymans stellte uns die Hamburger $\mathrm{Fa}$. „Holzimprägnierung Bauisolierung Nordwest" Fruchtkörper zur Verfügung. Die Bestrahlung der Sporen erfolgte in einer Suspension mit der Keimflüssigkeit folgender Zusammensetzung: I $\%$ Zitronensäure $+100 \mu \mathrm{g} /$ 
I00 ml Vit. B (Czaja 1960). Als Quelle für die Bestrahlung der Sporen stand eine $10 \mathrm{kci}{ }^{60} \mathrm{Co}$-Bestrahlungsanlage mit einer Dosisleistung von 4,I $\times 10^{5} \mathrm{rad} / \mathrm{h}$ zur Verfügung. Zur Keimung der Sporen verwendeten wir sog. Böttcher'sche Kammern. Es erwies sich für die Keimungsrate als vorteilhaft, die Sporensuspension auf einer reinen, dünnen Agarschicht auf dem Deckglas aufzutragen. Auf diese Weise konnte auch die gleichmäßige Verteilung der Sporen gewährleistet werden. Die prozentuale Keimungsrate wurde nach Auszählung von etwa 300 Sporen je Probe in vierfacher Wiederholung ermittelt.

\section{Ergebnisse}

\section{a) Merulius lacrymans}

\section{Bestrahlung im Biomalz-Agar}

Die Bestrahlungsversuche im Biomalz-Agar ergaben, daß Merulius lacrymans bereits auf eine geringfügige Erhöhung der Temperatur während der Bestrahlung reagiert. Dies hängt aller Wahrscheinlichkeit nach mit dem für diese Pilzart so charakteristischen engen Temperaturoptimum zusammen. Tabelle I enthält die Ergebnisse der Bestrahlungsversuche im BiomalzAgar. Die Zahlen drücken das prozentuale Längenwachstum des Oberflächenmycels aus. Sie wurden nach 7 Tagen Wachstumszeit durch Messung des Durchmessers der gebildeten Kolonien in mm ermittelt. Der maximale, relative Fehler der angegebenen Prozentwerte beträgt im oberen Bereich (bis I5\% Mycelwachstum) $\pm 5 \%$, bei kleineren Wachstumsraten $\pm 15 \%$ des jeweiligen Mittelwertes.

\section{Tabelle I}

Prozentuales Mycelwachstum von Merulius lacrymans nach Bestrahlung im Biomalz-Agar. Kontrolle bei $20^{\circ} \mathrm{C}=100 \%$

\begin{tabular}{c|c|c|c}
\hline D [krad] & $20^{\circ} \mathrm{C}$ & $30^{\circ} \mathrm{C}$ & $32^{\circ} \mathrm{C}$ \\
\hline 0 & 100 & 20 & 15 \\
0,21 & 62 & 15 & 9 \\
0,64 & 101 & 19 & 14 \\
2,1 & 77 & 23 & 12 \\
6,4 & 46 & 4 & - \\
21,0 & 22 & 1 & - \\
64,0 & 5 & - & - \\
210,0 & 1 & - &
\end{tabular}

Aus der Tabelle ist als erstes zu entnehmen, daß das Längenwachstum mit erhöhter Strahlendosis im großen und ganzen kontinuierlich abnimmt. Die Abnahme der Strahlenresistenz bei höheren Temperaturen ist eindeutig. Während für die Abtötung des Mycels bei $20^{\circ} \mathrm{C}$ eine Dosis von mehr als 2 ro krad benötigt wird, läßt sich die gleiche Strahlenwirkung bei $32^{\circ} \mathrm{C}$ bereits durch etwa $6 \mathrm{krad}$ erreichen. Die z. T. beobachtete Wiederzunahme des Wachstums mit steigender Strahlendosis ist nichts Ungewöhnliches. Deratige Phänomene sind an höheren Pflanzen vielfach beobachtet (Bayer. Ldw. Jahrb. I965, IAEA-Reports 1966). Sie können nicht schlechthin als Stimulation betrachtet werden, sondern haben häufig den Charakter pathologischer Prozesse. Zur Klärung müßten sehr viel mehr Kriterien der Strahlenwirkung als nur eines, nämlich das Mycelwachstum herangezogen werden (Glubrecht 1965). Bei der praktischen Zielsetzung dieser Arbeit genügt aber das Ergebnis, welche Strahlendosen bei den verschiedenen Temperaturbedingungen das Wachstum ganz zum Erliegen bringen.

\section{Bestrahlung im $\mathrm{Holz}$}

Über die Strahlenresistenz von Merulius läßt sich erst dann eine endgültige Aussage von praktischem Wert machen, wenn die Bestrahlung unter natürlichen Bedingungen durchgeführt wird. Ein wesentlicher Punkt ist dabei die Temperaturabhängigkeit. Wir bestrahlten daher Merulius-infiziertes Holz bei unterschiedlichen Temperaturen. Aus neueren Arbeiten ist bekannt, daß bei Bestrahlungstemperaturen um den Gefrierpunkt bei Sporen von Clostridium botulinum gute Ergebnisse hinsichtlich der Abnahme der Strahlenresistenz erzielt wurden. Dabei wird angenommen, daß sich bei Bestrahlungen in eingefrorenem Zustand beim Übergang in die flüssige Phase durch die Erhöhung der Diffusionsrate der erzeugten Radikale die Resistenz verringert, um danach zunächst wieder anzusteigen (Grecz 1966). Daher untersuchten : wir Merulius lacrymans auch bei einer Bestrahlungstemperatur von $0^{\circ} \mathrm{C}$. Das Ergebnis dieser Versuche zeigt Tab. 2.

Tabelle 2

Prozentuales Längenwachstum des Mycels von Merulius lacrymans nach Bestrahlung im Holz. Kontrolle bei $20^{\circ} \mathrm{C}=100 \%$ (Fehlerangaben s. a) $\mathrm{r}$.)

\begin{tabular}{c|r|r|r|r|r}
\hline D[kra 1] & $20^{\circ} \mathrm{C}$ & $26^{\circ} \mathrm{C}$ & $28^{\circ} \mathrm{C}$ & $30^{\circ} \mathrm{C}$ & $0^{\circ} \mathrm{C}$ \\
\hline 0 & 100 & 12 & 48 & 7 & 105 \\
$0,2 \mathrm{I}$ & 68 & 3 & 25 & - & \\
0,64 & 25 & 2 & 8 & - & 112 \\
$2, \mathrm{I}$ & 14 & 28 & 7 & - & 54 \\
6,4 & 5 & 6 & 3 & - & 12 \\
21,0 & 1 & - & 1 & - & - \\
64,0 & - & - & - & - & - \\
210,0 & - & - & - & - &
\end{tabular}

Die wichtigste Erkenntnis nach diesen Versuchen ist, daß Merulius im Holz bereits bei niedrigeren Strahlendosen das Wachstum einstellt, als es bei der Bestrahlung im Biomalz-Agar der Fall ist. Die Ursache hierfür liegt vermutlich in der physiologisch günstigeren Zusammensetzung des Biomalzes. Außerdem handelt es sich bei den Holzproben hauptsächlich um Substratmycel im Gegensatz zum Biomalz, wo auch Oberflächenmycel in größeren Mengen in den Bestrahlungsproben vorkommt. Für die praktische Bekämpfung ist von Bedeutung, daß das Mycel bei $26^{\circ} \mathrm{C}$ bereits nach der Applizierung einer Dosis von etwa $20 \mathrm{krad}$ völlig abstirbt. Bei dem Wiederanstieg der Resistenz bei $28^{\circ} \mathrm{C}$ werden wir es wohl mit einem ähnlichen Effekt zu tun haben, wie dies in den mittleren Bereichen der Strahlendosis der Fall ist (s. vorhergehender Abschnitt). Es wird im allgemeinen angenommen, daß Temperaturen von $40^{\circ} \mathrm{C}$ für Merulius letal sind. Dieser Effekt ist jedoch stark von der Dauer der Temperatureinwirkung abhängig und die hierbei gewonnenen Werte haben so große Schwankungen, daß die „Abtötung“ durch Temperaturerhöhung nicht mit Sicherheit als irreversibel bezeichnet werden kann. Kaum ein anderer Pilz weist in seinem Wachstum so große Schwankungen wie Merulius auf. Deshalb wurden zur Sicherung der Ergebnisse die einzelnen Behandlungen sehr oft wiederholt. Die einzelnen Ergebnisse in der Tabelle stellen jeweils das Mittel von 30 Versuchsgliedern dar. Die Fehlerwerte liegen wie oben angegeben. 


\section{Bestrahlung der Sporen von Merulius lacrymans}

Anfangs wurde schon erwähnt, daß die Sporen an der räumlichen Verbreitung dieses Pilzes beteiligt sind, und zwar besonders bei größeren Entfernungen. Allein deswegen ist eine Untersuchung gerechtfertigt, zumal die Erkundung der Strahlenresistenz der Basidiosporen auch in allgemein-biologischer Hinsicht von Interesse sein kann.

$\mathrm{Da}$ die Sporengewinnung im Laboratorium sehr problematisch ist und auch im Fachschrifttum wenig darüber bekannt ist, gewannen wir unser Sporenmaterial aus natürlichen Quellen (zur Lippe u. Nesemann 1959). Der Tatsache, daß Sporen unterschiedlichen Alters auch eine unterschiedliche Strahlenresistenz aufweisen, konnte dadurch Rechnung getragen werden, daß für die Bestrahlungsversuche Fruchtkörper verschiedenen Alters verwendet wurden. Im allgemeinen konnte eine 50-60\%ige Keimungsrate erzielt werden. Die Prüfung der Strahlenresistenz der Sporen erfolgte bei verschieden hohen Temperaturen in den $\mathrm{Ab}$ stufungen $20^{\circ} \mathrm{C}, 26^{\circ} \mathrm{C}, 28^{\circ} \mathrm{C}, 35^{\circ} \mathrm{C}$ und $0^{\circ} \mathrm{C}$. Die Tabelle 3 zeigt die Ergebnisse der Bestrahlungsversuche mit Sporen.

Tabelle 3

Die prozentuale Keimungsrate von Merulius-Sporen nach Bestrahlung. Kontrolle bei $20^{\circ} \mathrm{C}=100 \%$

\begin{tabular}{|c|c|c|c|c|c|}
\hline [krad] & $20^{\circ} \mathrm{C}$ & $26^{\circ} \mathrm{C}$ & $28^{\circ} \mathrm{C}$ & $35^{\circ} \mathrm{C}$ & $0^{\circ} \mathrm{C}$ \\
\hline 0 & 100 & 3I & 75 & I7 & 68 \\
\hline 0,64 & 90 & 38 & 77 & & \\
\hline $2, I$ & 76 & 36 & 68 & 15 & 64 \\
\hline 6,4 & 46 & 32 & 27 & 20 & 67 \\
\hline 21,0 & 32 & 9 & 40 & 21 & 97 \\
\hline 64,0 & 9 & 58 & IO & 8 & 43 \\
\hline 210,0 & I & 3 & - & - & 17 \\
\hline 640,0 & - & & & & 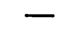 \\
\hline
\end{tabular}

Aus diesen Daten ist zu ersehen, daß die Sporen eine höhere Resistenz gegenüber ionisierenden Strahlen aufweisen als das Mycel. Zur völligen Verhinderung der Keimung ist die Applizierung einer Dosis von $6 \cdot 10^{5} \mathrm{rad}$ bei Zimmertemperatur notwendig. Eine Erhöhung der Temperatur wirkt sich bei den Sporen nicht in dem Maße auf die Strahlensensibilität aus, wie dies beim Mycel der Fall war. Eine Erhöhung der Strahlenwirkung bei der Bestrahlung bei $0^{\circ} \mathrm{C}$ ist nicht eingetreten. Durch den ruhenden Zustand sind die Sporen offensichtlich auch bei relativ ungünstigen Lebensbedingungen zum Überleben befähigt, da ihre physiologische Tätigkeit auf ein Minimum reduziert ist. Diese Tatsache spiegelt sich auch in der höheren Strahlenresistenz wieder. Immerhin erniedrigt sich die tödliche Strahlendosis bei $28^{0} \mathrm{C}$ auf 2,I $\times 10^{5} \mathrm{rad}$. Diese Dosis kann auch bei $35^{\circ} \mathrm{C}$ nicht unterschritten werden.

\section{Spezielle Untersuchungen am Mycel}

Im Hinblick auf die praktische Bekämpfung eines Pilzbefalles in Gebäuden befaßten wir uns mit Fragen; deren Lösung zur Erleichterung der technischen Durchführung beitragen soll.

Die Frage, ob der Pilz, der eine Mauer durchwachsen hat, auch . ohne Holz als Nahrungsquelle weiterwachsen kann, konnte durch einen einfachen Versuch schnell beantwortet werden. In einem Mauerwerk in kleinem Maßstab, das von dem angrenzenden infizierten $\mathrm{Holz}$ aus befallen war, wuchs das Mycel nicht mehr weiter, sobald die Verbindung zum Holz unterbunden wurde. Das Mycel blieb jedoch im Mauer- werk weiter lebensfähig, was dadurch bewiesen werden konnte, daß noch nach einer viermonatigen Ruhezeit gesundes Holz vom Mauerwerk aus infiziert wurde.

In dem vorigen Versuch wurde die Verbindung zwischen dem Holz und der Wachstumszone durch das mechanische Zerstören der Mycelstränge aufgehoben. Wenn der gleiche Effekt durch eine partielle Bestrahlung des Mycels erzielt werden könnte, wobei die Nahrungs- und Flüssigkeitsleitung nicht durch mechanische Zerstörung, sondern durch Strahleneinwirkung außer Funktion gesetzt wird, würde dies bei der praktischen Bekämpfung bedeuten, daß die Bestrahlung ganzer Wandflächen $u$. U. eingespart werden könnte. Selbstverständlich dürfte eine Bekämpfung des Infektionsherdes des Pilzes nicht unterlassen werden. Mit Hilfe eines Versuches, dessen Anordnung auf Abb. 5 schematisch dargestellt ist, sollte diese Frage beantwortet werden.
A

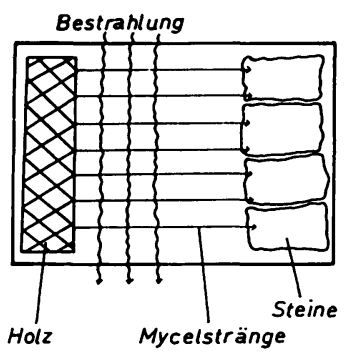

$B$

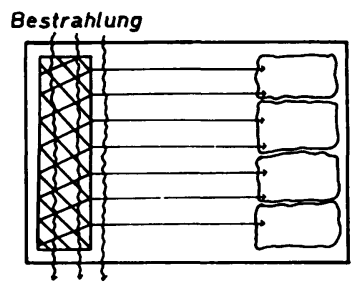

Abb. 5. Schematische Versuchsanordnung zur Bestrahlung des Mycels von Merulius lacrymans

Für diesen Versuch wurden gleich große mit Mycel durchwachsene Holzstücke auf eine Glasplatte gelegt und ihnen gegenüber Mauersteine in einer Entfernung von etwa $15 \mathrm{~cm}$ aufgebaut. Bei der Anordnung $A$ wird nur das Mycel quer an einer Stelle bestrahlt, während bei Anordnung B das Holz und ein Teil des herausgewachsenen Mycels bestrahlt wird. Als Bestrahlungsquelle diente eine ${ }^{60} \mathrm{Co}$-Anlage mit einer Aktivität von Io $\mathrm{kCi}$. Die Bestrahlung wurde bei Zimmertemperatur vorgenommen, sobald das Mycelwachstum die Steine erreichte. Es wurde eine Dosis von $2 \cdot 10^{5} \mathrm{rad}$ appliziert. Das ist eine Letaldosis für das Mycel von Merulius, jedoch werden dabei am Mycel keine äußerlich wahrnehmbaren Veränderungen verursacht.

Nach der Bestrahlung der beiden Anordnungen stellte der Pilz sein Wachstum zunächst ein. In Anordnung $A$ jedoch, in der das Mycel im Holz nicht abgetötet war, wuchs es nach einiger Zeit wieder nach. In Anordnung B, in der auch der Befallsherd beseitigt worden war, zeigte sich kein erneutes Wachstum. So bestätigte sich die Erwartung, daß dem Mycel durch Bestrahlung die Fähigkeit verlorengeht, Nahrung und Wasser zu den Angriffsstellen zu leiten. Da bei unseren Untersuchungen die praktische Bekämpfung im Vordergrund stand, wurde auf eine weitere Untersuchung dieser Erscheinung verzichtet.

\section{Beobachtungen am strahlengeschädigten Mycel}

Bei der Ausführung der Bestrahlungsversuche mit Merulius sind wir auf einige charakteristische Erscheinungen aufmerksam geworden, die hier kurz beschrieben werden sollen. Bereits subletale Strahlendosen riefen eine makroskopisch erkennbare Veränderung im Mycelwachstum des Pilzes hervor. Bei diesen Strahlendosen zerfällt in den Kolbenkulturen das einheitliche Wachstumszentrum in mehrere kleine Zentren, wie dies aus der Abb. 6 ersichtlich wird.

Hierbei zeigt das Oberflächenmycel nicht mehr die langgestreckte Form, sondern es ist eher kurz und schimmelartig. Die weiße Farbe bleibt jedoch erhalten. Bei einer mikroskopischen Betrachtung sind zahlreiche Gemmen erkennbar, die sich aus dem zerfallenen Mycel gebildet haben. Solche Gemmen zeigt die Abb. 7.

Bei Kulturen auf Biomalz-Agar konnte diese Erscheinung ebenfalls registriert werden. Nach dem Übertragen von Impfstücken auf neuen Biomalz-Agar blieb diese Wuchsform und die Gemmenbildung über mehr als 4 Generationen hindurch erhalten. Wurde dagegen $\mathrm{Holz}$ mit diesem Mycel infiziert, zeigte sich wieder ein normales Wachstum, gekennzeichnet durch kräftiges Oberflächenmycel mit langen Hyphen. Es lag durch kräftiges Oberflächenmycel mit langen Hyphen. 


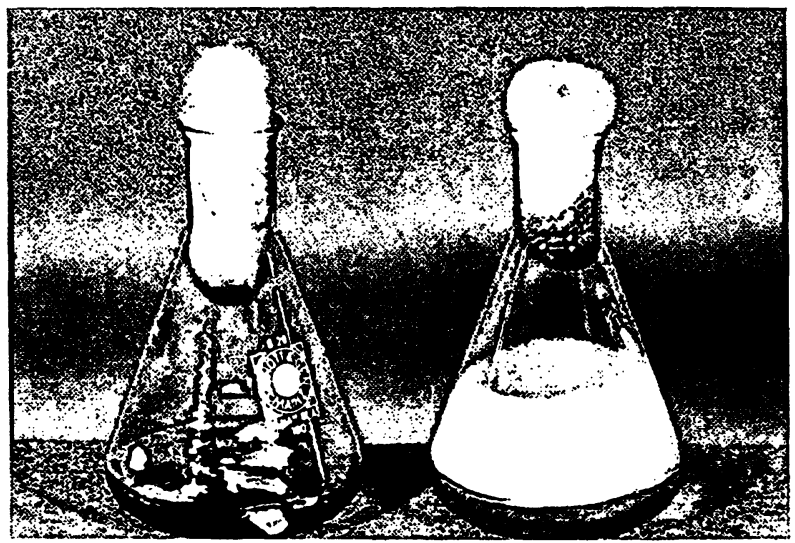

Abb. 6. Bestrahlte und unbestrahlte Kulturen von Merulius in einer Nährlösung nach Lyr

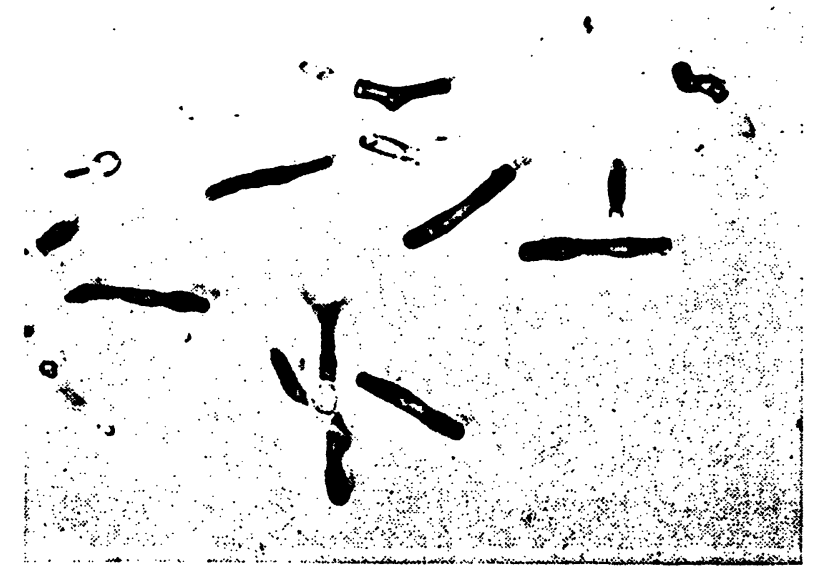

Abb. 7. Durch Bestrahlung verursachte Gemmenbildung

daher die Vermutung nahe, daß es sich hierbei um eine sekundäre Strahlenwirkung handelt. Der Pilz muß die Fähigkeit verloren haben, bestimmte lebenswichtige Nährstoffe zu synthetisieren. Um festzustellen, um welche Stoffe bzw. um welchen Stoff es sich hierbei handelt, wurde das geschädigte Mycel von Merulius auf Komplett- und Minimalnährboden mit dem Zusatz von verschiedenen Aminosäuren getestet. Der Minimalnährboden war folgendermaßen zusammengesetzt:

\begin{tabular}{lrlr}
$\mathrm{NaNO}_{3}$ & $2 \mathrm{~g}$ & $\mathrm{FeSO}_{4}$ & \multicolumn{1}{c}{$\mathrm{Spur}_{3}$} \\
$\mathrm{KH}_{2} \mathrm{PO}_{4}$ & $\mathrm{Ig}$ & $\mathrm{Glucose}$ & $30 \mathrm{~g}$ \\
$\mathrm{KCl}$ & $0,5 \mathrm{~g}$ & Difcoagar & $20 \mathrm{~g}$ \\
$\mathrm{MgSO}_{4} \cdot 7 \mathrm{H}_{2} \mathrm{O}$ & $0,5 \mathrm{~g}$ & Aqua dest. & $1000 \mathrm{ml}$
\end{tabular}

Der Komplettnährboden enthielt zusätzlich folgende Nährstoffe:

$2 \mathrm{~g}$ Pepton, 2,5 g Hefeextrakt, I ml Vitamin-Lösung, Io $\mathrm{ml}$ Aminosäurelösung.

Die Aminosäurelösung enthielt jeweils $2 \mathrm{~g}$ der jeweiligen Aminosäure, gelöst in $100 \mathrm{ml} \mathrm{Wasser}$. Auch die zu dem Minimalnährboden zugesetzten Aminosäuren hatten die gleiche Konzentration.

In Tabelle 4 sind diese Aminosäuren aufgezählt und das Ergebnis ist angegeben. Es bedeuten:

$$
\begin{aligned}
& -\quad \text { Schädigung nicht aufgehoben } \\
& +\quad \text { Schädigung zum Teil aufgehoben } \\
& ++ \text { Schädigung vollständig aufgehoben }
\end{aligned}
$$

Wie aus den Ergebnissen der Tabelle hervorgeht, eignet sich Asparagin am besten für die Aufhebung der Strahlenschädigung. Es kommt bei einigen anderen Aminosäuren ebenfalls zu geringen Wachstumssteigerungen. Ob jedoch eine erhöhte Konzentration dieser Stoffe zu dem erwarteten Ergebnis führen würde, müßte noch geprüft werden.
Tabelle 4

Wachstum des geschädigten Mycels von Merulius lacry-

\begin{tabular}{|c|c|}
\hline Nährboden & Ergebnis \\
\hline 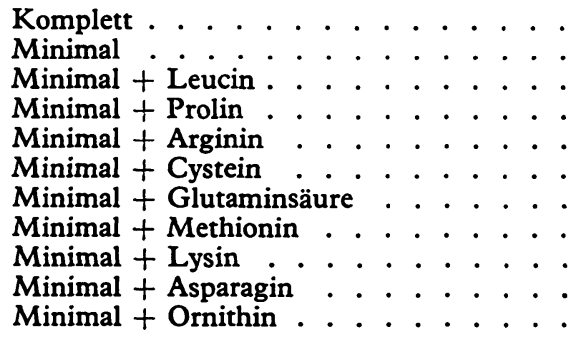 & $\begin{array}{l}\frac{+}{-} \\
\frac{-}{+} \\
\frac{+}{+} \\
\frac{+}{+t} \\
+\end{array}$ \\
\hline
\end{tabular}
mans auf Komplett-u. Minimalnährboden mit Aminosäurezusätzen

\section{b) Coniophora cerebella}

Coniophora cerebella hat gegenüber Nadel- und Laubholz ungefähr die gleiche Zersetzungskraft wie Merulius lacrymans. Coniophora bevorzugt allerdings im Gegensatz zu Merulius eine höhere relative Luftfeuchtigkeit. Diese Voraussetzung ist in neuerer Zeit besonders gegeben, wenn bei Baumaßnahmen nicht voll ausgetrocknetes Holz verwendet wird. So darf man Coniophora cerebella als typischen ,Neubaupilz" charakterisieren. Er unterscheidet sich außerdem von Merulius durch seine Temperaturansprüche. Die maximale Wachstumstemperatur liegt bei Coniophora bedeutend höher, daher war von besonderem Interesse festzustellen, bei welcher Temperatur der Pilz in einen $\mathrm{Zu}-$ stand höherer Strahlenempfindlichkeit gebracht werden kann.

\section{Bestrahlung im Biomalz-Agar}

Die Ergebnisse der Bestrahlungsversuche im Biomalz-Agar sind in der Tabelle 5 zusammengestellt. Die Zahlen stellen das durchschnittliche Wachstum des Oberflächenmycels dar, und zwar nach 5 Tagen Kulturzeit im Anschluß an die Bestrahlung. Die Versuche wurden in glcicher Weise wie bei Merulius durchgeführt.

Tabelle 5

Prozentuales Mycelwachstum von Coniophora cerebella nach Bestrahlung im Biomalz-Agar. Kontrolle. bei $20^{\circ} \mathrm{C}=100 \%$

\begin{tabular}{c|c|c|c}
\hline $\mathrm{D}$ [krad] & $20^{\circ} \mathrm{C}$ & $34^{\circ} \mathrm{C}$ & $36^{\circ} \mathrm{C}$ \\
\hline 0 & 100 & 68 & 37 \\
$0,2 \mathrm{I}$ & 92 & 88 & 25 \\
0,64 & 106 & 66 & 47 \\
$2, \mathrm{I}$ & 92 & 92 & $\mathrm{I}$ \\
6,4 & 82 & $5 \mathrm{I}$ & 2 \\
21,0 & $5 \mathrm{I}$ & - & - \\
64,0 & 7 & - & - \\
210,0 & - & &
\end{tabular}

Aus den Daten der Tabelle ist zu ersehen, daß Coniophora cerebella etwa die gleiche Strahlenresistenz wie Merulius lacrymans bei Bestrahlung bei Zimmertemperatur aufweist, nämlich völlige Aufhebung des Wachstums bei $20^{\circ} \mathrm{C}$ mit $200 \mathrm{krad}$. Auffällig ist, daß zur Erniedrigung der letalen Strahlendosen viel höhere Temperaturen erforderlich sind, als dies bei Merulius der Fall war. Mit $20 \mathrm{krad}$ wird der gleiche letale Effekt bei Merulius bei $26^{\circ} \mathrm{C}$ erzielt, bei Coniophora dagegen erst bei $34^{\circ} \mathrm{C}$. Eine Abtötung des Mycels allein durch 
Temperaturerhöhung im technisch realisierbaren Maßstab scheint hier noch wesentlich aussichtsloser als bei Merulius.

\section{Bestrahlung im Holz}

Inwieweit sich bei Coniophora cerebella die Strahlenresistenz bei Bestrahlung im Kiefernholz im Gegensatz zum Biomalz-Agar ändert, kann aus Tabelle 6 entnommen werden.

Tabelle 6

Prozentuales Mycelwachstum von Coniophora cerebella nach Bestrahlung im Holz. Kontrolle bei $20^{\circ} \mathrm{C}=100^{\circ} ;$

\begin{tabular}{|c|c|c|c|}
\hline$D$ [krad] & $20^{\circ} \mathrm{C}$ & $34^{\circ} \mathrm{C}$ & $36^{\circ} \mathrm{C}$ \\
\hline 0 & 100 & 43 & 77 \\
\hline $0,2 \mathrm{I}$ & II 2 & 36 & 46 \\
\hline 0,64 & 104 & 54 & 67 \\
\hline 2,1 & 84 & 12 & 48 \\
\hline 6,4 & 62 & 2 & 4 \\
\hline 21,0 & 17 & - & - \\
\hline 64,0 & - & - & - \\
\hline 210,0 & - & - & - \\
\hline
\end{tabular}

Die Ergebnisse zeigen, daß auch Coniophora cerebella ähnlich wie Merulius lacrymans auf die Standortveränderung mit einer wesentlichen Abnahme der Strahlenresistenz reagiert. Die Tendenz der Sensibilisierung bei erhöhter Bestrahlungstemperatur bleibt auch im Holz erhalten. Zur völligen Abtötung des Mycels von Coniophora cerebella genügen Strahlendosen von 20 krad bei gleichzeitiger Erhöhung der Temperatur auf $34^{\circ} \mathrm{C}$.

\section{Diskussion}

Die Ergebnisse der hier dargestellten Untersuchungen können eine Basis für die Bekämpfung holzzerstörender Pilze mit Hilfe von ionisierenden Strahlen bieten. Die Grundlage dafür ist die auffallende Temperaturabhängigkeit der Strahlenresistenz beider hier untersuchter Pilzarten. Dabei ist es offenbar nicht möglich, die Strahlenwirkung vollständig durch eine Erhöhung der Temperatur zu ersetzen. Ganz abgesehen von den technischen Schwierigkeiten und der großen Unsicherheit, die eine Bekämpfung nur durch Erwärmung der befallenen Holz- und Gebäudeteile bedeuten würde, liegt auch offensichtlich kein reiner Synergismus zwischen Strahlen- und Temperaturwirkung vor. Das zeigt eindeutig die bei der Bestrahlung des Pilzmycels immer wieder beobachtete Rückläufigkeit der Wirkung in einem bestimmten Dosisbereich (s. Tabelle I und Tabelle 5 bei Dosen von $0,64 \mathrm{krad}$ ). Offenbar führen kleinere Strahlendosen zu ganz anderen Wirkungen als höhere. Oberhalb von etwa $5 \mathrm{krad}$ scheinen die Strahlenwirkungen dann definitiv irreversibel zu sein. Bei höheren Temperaturen verlagert sich dieser Bereich offenbar $\mathrm{zu}$ niedrigeren Strahlendosen. Es ist auffallend, daß vergleichbare Effekte bei der Sporenbestrahlung (Tabelle 3) bei $20^{\circ} \mathrm{C}$ überhaupt nicht und bei anderen Temperaturen in anderen Dosisbereichen auftreten.

$\mathrm{Da}$ die vorliegende Arbeit in erster Linie die Anwendung von Bestrahlungsmethoden für die praktische Bekämpfung von Holzschädlingen klären sollte, erübrigt es sich, die ungewöhnlichen Resultate bei der Bestrahlung mit kleineren Dosen, die nicht zur völligen
Eliminierung des Mycelwachstums und der Sporenkeimung führen, hier näher zu erörtern. Auch müßten dazu vermutlich sehr viel umfangreichere Untersuchungen angestellt werden. Entscheidend ist, daß der Dosisbereich, in dem die irreversible Schädigung bei technisch noch durchaus erzeugbaren Temperaturen erfolgt, einen praktischen Einsatz von Bestrahlungsmethoden zur Bekämpfung der Holzschädlinge zuläßt.

Werden bei der Bekämpfung auch die Sporen berücksichtigt, so kann u. U. ihre geringere Strahlenempfindlichkeit Schwierigkeiten mit sich bringen. Allerdings ist es ja bekannt, daß die Sporen vornehmlich an den Wandoberflächen zu finden sind, wo bei jeder Art von Bestrahlungstechnik mit höheren Dosen als im Inneren gerechnet werden kann. Wird dabei berücksichtigt, daß beim Vorkommen von Coniophora ohnehin die Temperaturen bei der Bestrahlung auf etwa $34^{\circ} \mathrm{C}$ erhöht werden müssen, so werden zwangsläufig auch die resistenteren Sporen mit erfaßt, die aber bei der unmittelbaren Verbreitung des Pilzes eine untergeordnete Rolle spielen.

In der Literatur liegen andererseits Ergebnisse vor, die zeigen, daß andere holzzerstörende Pilzarten wie Poria vaporaria und Lentinus lepidus in ihrer Strahlenund Hitzeresistenz Coniophora ähnlich sind, so daß eine kombinierte Behandlung auch hier zum Ziele führen kann. In jedem Falle würden die in Frage kommenden Dosen auch ausreichen, holzzerstörende Insekten abzutöten. Es wurde oben bereits darauf hingewiesen, daß die Abmessungen der Proben entsprechend der „Klötzchenmethode" aus bestrahlungstechnischen Gründen nicht eingehalten werden konnten (Liese, Nowak, Peters und Rabanus I935). Aus Arbeiten mit Mikroorganismen wurde bekannt, daß die Konzentration der bestrahlten Organismen einen Einfluß auf die Strahlenresistenz hat. Die Konzentration des Mycels im Holz ist aber weitgehend unabhängig von der Größe des befallenen Holzstückes, so daß in diesem Fall die Ergebnisse für die Gesamtvolumina representativ sind.

Beim Vergleich dieser Methode mit der chemischen Behandlung können schon jetzt einige Vorteile hervorgehoben werden: Ionisierende Strahlen wirken nicht selektiv auf die Organismen wie eine große Zahl von Chemikalien, vorausgesetzt, es werden die notwendigen Dosen appliziert. Bei der Bestrahlung wird das ganze Volumen ohne bauliche Maßnahmen erfaßt. Chemische Schutzflüssigkeiten dagegen erreichen oft die Poren und Spalten im Mauerwerk unvollständig. Sofern diese unbehandelten Stellen mit Mycel durchsetzt sind, bilden sie den Ausgangspunkt neuer Infektion (Buro 1958). Aus der Sicht der Strahlenbekämpfung ist demnach die Vernichtung der Pilze mit chemischen Mitteln unzureichend. Eine sinnvolle gegenseitige Ergänzung beider Methoden zeigt sich indessen darin, daß nach einer Strahlenbehandlung die chemische Methode rein prophylaktisch herangezogen werden sollte.

Wie jedoch die gewonnenen grundsätzlichen Resultate für eine praktische Bekämpfung holzzerstörender Pilze im einzelnen anzuwenden sind, muß weiteren Untersuchungen und einer weiteren Arbeit vorbehalten bleiben. Handelt es sich um transportable Gegenstände, beispielsweise kunsthistorische Werte, so können diese in jeder geeigneten Bestrahlungsanlage 
unter Berücksichtigung der erhaltenen Ergebnisse von Schädlingen befreit werden, ohne daß es besonderer Maßnahmen oder zusätzlicher Versuche bedarf.

\section{Zusammenfassung}

Es wurden die Strahlenresistenz von Merulius lacrymans und Coniophora cerebella untersucht. Wir bestrahlten das Mycel der beiden Pilze sowie die Sporen von Merulius. Als Kriterium der Strahlenwirkung wurde das Mycelwachstum gemessen, bzw. die Keimungsrate der Sporen ermittelt.

Es hat sich gezeigt, daß eine Erhöhung der Bestrahlungstemperatur eine wesentliche Abnahme der Strahlenresistenz zur Folge hat. So sinkt die Letaldosis des Mycels von $200 \mathrm{krad}$ auf $20-30 \mathrm{krad}$ bei einer Erhöhung der Temperatur von $20^{\circ} \mathrm{C}$ auf $26^{\circ} \mathrm{C}$ (Merulius), bzw. auf $34^{\circ} \mathrm{C}$ (Coniophora). Die Sporen von Merulius reagieren nicht in gleicher Weise auf die Temperaturerhöhung. Zur völligen Verhinderung der Sporenkeimung werden auch bei erhöhter Temperatur Strahlendosen von etwa $600 \mathrm{krad}$ benötigt. Es wurde beobachtet, daß letale Strahlendosen die Leitfähigkeit des Mycels für Wasser und Nährstoffe zerstören. Die Möglichkeit der Beseitigung eines Pilzbefalles in Gebäuden mit Hilfe von ionisierenden Strahlen wird diskutiert.

Investigations on Radiation Resistance of Wood-Destroying Fungi

\section{Summary}

The radiation resistance of Merulius lacrymans and Coniophora cerebella was investigated. The mycelium of both fungi and the spores of Merulius were radiated. As criterion for the radiation effectiveness the mycelium growth was measured and the germination rate determined respectively.

It was shown that an increase of the radiation temperature results in a considerbale decrease of the radiation resistance. Thus the lethal dose of the mycelium decreases from $200 \mathrm{krad}$ to $20-30 \mathrm{krad}$ with a temperature increase from $20^{\circ} \mathrm{C}$ to $26^{\circ} \mathrm{C}$ (Merulius) to $34^{\circ} \mathrm{C}$ (Coniophora) respectively. The spores of Merulius do not react in the same way on temperature increase. For a complete prevention of a spore germination even at elevated temperatures radiation doses of $600 \mathrm{krad}$ are necessary. It was found that radiation doses ruin the water and nutrient conductivity of the mycelium. The possibility of curing fungi attack in buildings by means of ionizing rays is discussed.

Für wertvolle Ratschläge bei den mikrobiologischen Untersuchungen haben wir Herrn Prof. Dr. G. M. Hof fmann, Hannover, besonders zu danken.

\section{Literatur}

Babos, M.: Über die relative Strahlenwiderstandsfähigkeit der Mycelien einiger holzzerstörender Pilzarten. Ann. hist.nat. Mus. Hung, 54, 185-189 (1962).

Bay. Landw. Jahrbuch 42, Sonderheft I (1965).

Buro, A.: Untersuchungen über die Schwammbekämpfung im Mauerwerk. Berliner Bauwirtschaft 9, 164-170 (1958).

Czaja, A. Th.: Ửber das Aufkommen des echten Hausschwammes (Merulius lacrymans var. domesticus [Pers.] Falck) und des braunen Kellerschwammes (Coniophora cerebella [Pers.] Duby) aus ihren Sporen an verbautem Holz. Angew. Botanik 33, 107-121 (1960).

Frejdin, A. S., Gorsin, S. N. u. Krapivna, J. G.: Die ionisierende Strahlung als sterilisierender Faktor. Sammelwerk: Fragen des Holzschutzes (Russisch). Moskva: Goslesbumisdat, I0I-I08 (I96I).

Glubrecht, H.: Zum Begriff der Testreaktion bei der biologischen Strahlenwirkung. Bay. Landw. Jahrb. 42, 7-10 (I965).

Grecz, N.: Theoretical and applied aspects of radiation Dvalues for spores of Clostridium botulinum. Proc. of IAEASymp. 1966, Karlsruhe, S. 307-320.

IAEA-Reports: Wien, Serie Nr. 64 (1966).

IAEA-Symp. Karlsruhe, Food Irradiation, I966.

Kreisel, H.: Die phytopathogenen Großpilze Deutschlands. VEB Gustav Fischer Verlag Jena (I96I).

Künzelmann, E.: Die Gefährdung mineralisch gebundener Holzwolle-Leichtbauplatten durch holzzerstörende Pilze. Holztechnologie 2, 225-228 (1961).

Liese, Novak, Peters u. Rabanus: Toximetrische Bestimmung von Holzkonservierungsmitteln. Angew. Chemie, Beihefte Nr. II (I935).

Lippe zur, T. u. Nesemann, G.: Über die Fruchtkörperbildung von Merulius lacrymans domesticus Falck. Arch. Mikrobiol. 34, 132-148 (1959).

Lyr, H.: Die Bildung von Ektoenzymen durch holzzerstörende und holzbewohnende Pilze auf verschiedenen Nährböden. I. Mitt.: Pectin als C-Quelle. Arch. Mikrobiol. 33, 266 (1959).

\title{
Möglichkeiten der Anwendung von Ultraschall bei der Untersuchung von Holz und Holzspanplatten
}

\author{
Von Helmut Becker ${ }^{\star}$ )
}

\section{Mitteilung aus dem Institut für Holzphysik und mechanische Technologie des Holzes der Bundes-} forschungsanstalt für Forst- und Holzwirtschaft in Reinbek

\section{Einleitung}

Bei der zerstörungsfreien Qualitätskontrolle von homogenen, isotropen Materialien stellt Ultraschall ein vielseitig einsetzbares Hilfsmittel dar, das sich insbesondere für die Identifizierung von örtlichen Fehlstellen außerordentlich bewährt hat. Es erhebt sich daher die Frage, inwieweit Ultraschall auch bei der zer-

*) Der Deutschen Gesellschaft für Holzforschung und der Arbeitsgemeinschaft Industrieller Forschungsvereinigungen danken wir für die finanzielle Unterstützung dieser Arbeit. störungsfreien Prüfung von Holz und Holzwerkstoffen eingesetzt werden kann. Das bei der Prüfung von Stahl verwendete Impuls-Echo-Verfahren ist bei Holz nicht anwendbar, da Holz für Ultraschall nur mäßig gut durchlässig und - wie es in der Fachsprache heißt nicht "schallhart" genug ist.

Der Schallwellenwiderstand des Holzes, der definiert ist als Produkt aus Dichte und Schallgeschwindigkeit $(R=\varrho \cdot v)$ unterscheidet sich - im Vergleich zu Stahl - nur mäßig von dem Schallwellenwiderstand der Luft. Das bedeutet, daß die Reflexion des Schalles Bereitgestellt von | Technische Informationsbibliothek Hannover 